\title{
Last Subject In Identity
}

National Cancer Institute

\section{Source}

National Cancer Institute. Last Subject In Identity. NCI Thesaurus. Code C142597.

The identity of the final subject to enroll in a clinical trial. 